\title{
ANÁLISIS MACROESTRUCTURAL DE PRESENTACIONES ACADÉMICAS ORALES EN ESPAÑOL L2
}

\author{
María Ángeles Vergara PADILla \\ Universidad Antonio de Nebrija (Madrid) \\ Irini MAVROU \\ Universidad Antonio de Nebrija (Madrid)
}

\section{RESUMEN}

Las exigencias del contexto universitario demandan el análisis y sistematización de los géneros textuales, escritos y orales. Esta circunstancia, unida a la importancia de atender a las necesidades de los estudiantes de educación terciaria, tanto en su lengua materna como en segundas lenguas, nos instan a la necesidad de agilizar la labor de caracterizar los textos académicos. Este estudio tiene como objetivo analizar la estructura global de 20 presentaciones académicas orales realizadas por estudiantes estadounidenses, aprendientes de español como segunda lengua, en contexto de inmersión en una universidad española. Fruto del análisis realizado, se ofrece una propuesta de la macroestructura de la presentación académica oral partiendo del modelo de Villar (2014).

PALABRAS CLAVE: presentación académica oral, macroestructura, géneros textuales, español como segunda lengua, contexto universitario.

\section{ABSTRACT}

The demands of the university context require the analysis and systematization of textual genres, both oral and written. Moreover, the importance of dealing with the needs of students of Tertiary Education urges us to speed up the task of characterizing the academic texts in first and second language. The aim of this study is to analyze the global structure of 20 academic oral presentations made by students from the United States. The participants were learners of Spanish as a second language in an immersion context at a Spanish university. As a result of the above analysis, this study offers a proposal of the global structure of academic oral presentations based on Villar's (2014) model.

KEYWORDS: academic oral presentation, macrostructure, textual genres, Spanish as a second language, university context. 


\section{INTRODUCCIÓN}

La oralidad en el ámbito académico es un concepto complejo y, a veces, difícil de definir con precisión. Como señala Mauranen (2007: 39):

La oralidad académica es exigente, compleja y, con frecuencia, los significados con un alto contenido abstracto requieren de una negociación al mismo tiempo que el discurso fluye. Los participantes deben estar preparados para gestionar el contenido intelectual de alto nivel de manera simultánea con otros actos de comunicación en tiempo real. La lengua de uso en el ámbito universitario es más sofisticada que en una interacción estereotipada, por ejemplo en transacciones de venta rutinarias o en situaciones comunicativas comunes en el ámbito del turismo. Los discursos en el ámbito de la educación superior incluyen también un amplio rango de variación en los niveles de formalidad y familiaridad. En este sentido, estos niveles ofrecen un material de investigación más rico y gratificante que otros intercambios comunicativos más sencillos.

La necesidad de indagar en el estudio sobre el funcionamiento de la oralidad -entendida como una competencia que debe desarrollarse junto con otras destrezas como la lectura y la escritura- a nivel universitario es una realidad que debe atenderse. Según Andrade-Calderón y Muñoz-Dagua (2014), es imprescindible investigar las dificultades de los estudiantes en lo que respecta a las tipologías textuales orales en el ámbito universitario. Muñoz-Dagua, Andrade-Calderón y Cisneros-Estupiñán (2015: 128), por su parte, opinan que la oralidad es decisiva para asimilar, confrontar y reelaborar el discurso académico sólido, que visibilice una educación terciaria de calidad. Estas autoras insisten en la importancia de revisar y avanzar en el análisis de los hábitos y actitudes que adoptan los estudiantes en sus intervenciones orales como parte de su proceso de acceso, comprensión e interpretación de las competencias propias de su formación disciplinar.

El presente estudio parte de la premisa de que los estudiantes internacionales que dedican un periodo de su formación superior a estudiar en un país extranjero deberían estar preparados para hacer frente a sus dificultades y carencias lingüísticas y comunicativas, por lo que las habilidades vinculadas a la expresión oral se han de reforzar en el aula universitaria no solo en la primera (L1) sino también en una segunda lengua (L2). De ahí el interés del presente estudio que persigue un doble objetivo: (a) analizar la estructura global (o macroestructura ${ }^{1}$ ) de las presentaciones acadé-

\footnotetext{
${ }^{1}$ Es necesario aclarar que los términos estructura global y macroestructura se utilizan en el presente estudio de manera indistinta para referirse a la organización jerarquizada y progresiva del tema de un texto, escrito u oral, presentado en ideas principales y secundarias que aportan significado al texto completo (van Dijk 1978).
} 
micas orales (PAO) en español como segunda lengua (EL2); y (b) ofrecer una propuesta de macroestructura de este género discursivo académico tomando como base de referencia el modelo de estructura global de la PAO de Villar (2014). En las siguientes líneas, se presenta una revisión breve de algunos conceptos clave relacionados con el género de la PAO.

\section{El CONCEPTO DE GÉNERO}

En el marco del Análisis de Discurso y de la Lingüística en general, el concepto de género ha sido definido de varias maneras, aunque todas parecen convergir. Según Swales (1990: 58):

Un género comprende un tipo de eventos comunicativos que comparten un conjunto de propósitos comunicativos. Estos propósitos son reconocidos por los miembros expertos de la comunidad discursiva en que se producen y constituyen, por tanto, el fundamento del género en cuestión. Este fundamento da forma a la estructura esquemática del discurso e influye y determina la selección del contenido y del estilo.

En el ámbito universitario, Rojas-García (2016: 190) considera que «el género es una posibilidad de hacer cosas con el lenguaje, siendo el aprendizaje una actividad psicológica, social pero especialmente lingüística». García-Izquierdo (2007: 121) comparte la idea de «la existencia de una estructura formal prototípica en los textos de especialidad que caracteriza hasta cierto punto los textos producidos en la comunicación especializada», así como el hecho de que los géneros discursivos son el resultado de una circunstancia específica de comunicación. La autora resalta dos rasgos del concepto de género:

1) su naturaleza dinámica, en el sentido de que se pueden producir cambios en los géneros dependiendo del ámbito de especialidad;

2) la no pertenencia del género a una categoría aislada, sino a un sistema en el que se establecen relaciones con los géneros de su propio ámbito y con géneros de otros ámbitos afines (Bazerman 1994, en García-Izquierdo 2007: 122-123).

Asimismo, considera imprescindible conocer cómo funcionan de manera contrastiva los géneros discursivos (escritos y orales) y establecer similitudes y diferencias en distintos niveles de análisis, tanto en la estructura externa como en la interna, de los textos académicos.

De las definiciones del concepto de género propuestas en la bibliografía especializada se puede apreciar una serie de rasgos comunes que caracterizan a los textos independientemente de su origen e idiosincrasia acadé- 
mica: los géneros son eventos comunicativos; poseen un propósito comunicativo y una función social predominante; tienen una estructura esquemática; hay tipos de textos que forman parte del género y que poseen unos patrones específicos en cuestiones de estructura, estilo, contenido y público; pueden llegar a ser prototípicos para una comunidad discursiva en un contexto sociocultural concreto.

Bajtín (1982) hizo la distinción entre géneros discursivos primarios y géneros discursivos secundarios. Los primarios son aquellos que surgen de la comunicación espontánea en diferentes situaciones, mientras que los secundarios se vinculan a un contexto de comunicación más marcado y formal, que puede pertenecer al ámbito literario, científico o ideológico. Ahora bien, es preciso señalar que no existe una delimitación clara que marque la frontera entre los géneros primarios y secundarios; por ejemplo, los géneros secundarios pueden llegar a transformar a los primarios cuando existe una escolarización de los géneros, es decir, cuando estos se llevan al aula (Dolz y Gagnon 2010). Otra clasificación es la propuesta por Ciapuscio (1994: 25), quien distingue entre género y tipos discursivos: el género lo relaciona con la dimensión histórico-cultural más general, mientras que los tipos discursivos con una dimensión estrictamente lingüística.

Desde el punto de vista del aprendizaje de las habilidades textuales y discursivas, los géneros pueden utilizarse como herramienta u objeto de enseñanza-aprendizaje en el ámbito académico-universitario tanto en la modalidad escrita como en la oral. Cuando una herramienta cultural como el género discursivo de la PAO -objeto del presente estudio- se convierte en una herramienta de aprendizaje, se habilita al estudiante para comprender la significación de dicho género y valerse de modelos de producción para practicarlo y acrecentar así su conciencia retórica discursiva del mismo.

\section{El DisCURSO ACADÉMICO ORAL}

El discurso académico circunscrito al ámbito universitario hace referencia al lenguaje utilizado por los miembros de la comunidad discursivoacadémica (profesores, instructores, coordinadores, discentes e investigadores) en los diferentes ámbitos vinculados a la realidad universitaria. En lo que respecta al caso concreto del discente, debe habituarse a lo largo de su periodo de formación terciaria al dominio del lenguaje académico, que pondrá en práctica en su futuro profesional y/o investigador. Se evidencia así el carácter profesional y científico que adquiere el lenguaje académico y, por ende, su carácter especializado, ya que habilita la comunicación entre diferentes actores (expertos, semilegos y legos) en las distintas áreas de estudio, investigación o futuro profesional. 
Tres autores que muestran la vinculación del discurso académico con el ámbito profesional y científico son Alcaraz Varó (2007) y Regueiro y Sáez (2013). Alcaraz Varó (2007) resalta el carácter interdisciplinar de las lenguas profesionales y académicas y los rasgos que lo caracterizan: el léxico, la morfosintaxis, el tipo de discurso predominante, la comunicación, los textos académicos y profesionales y el marco cultural diferenciado. Regueiro y Sáez (2013: 46-51), por su parte, concretizan las cuatro propiedades que todo discurso académico debería poseer, ya sea oral o escrito: adecuación, coherencia, cohesión y corrección.

El discurso utilizado en el ámbito universitario también se caracteriza por un estilo académico concreto, que puede determinar el lenguaje con mayor o menor grado de subjetividad, dependiendo de si adquiere un estilo especializado, divulgativo o formativo. Este último estilo está vinculado al aula y es el que constituye el objeto del presente estudio.

\section{LA PRESENTACIÓN ACADÉMICA ORAL}

La presentación académica oral (PAO) es considerara por Villar (2014: 43) como «un instrumento importante y presente en la vida académica utilizado con fines de evaluación (formativa o sumativa) en las certificaciones E/LE específicas y en las asignaturas universitarias de español en universidades europeas». Villar y Ainciburu (2013) puntualizan que la PAO es un género versátil, que sabe adaptarse a diferentes ámbitos tanto universitarios científicos como profesionales. Este rasgo le brinda la ventaja de ser un género textual dinámico, lo que permite afirmar que la PAO sabe amoldar su estilo académico a la intencionalidad comunicativa del contexto en el que se ubica, pudiendo llegar a adquirir un carácter tanto científico y objetivo como divulgativo y más subjetivo. Teniendo en cuenta la naturaleza dinámica del estilo académico, Vázquez (2016: 188) identifica dos tipos de PAO, aquellas vinculadas al ámbito universitario-escolar y las PAO relacionadas con el ámbito científico-académico; las primeras poseerían un estilo formativo, mientras que las segundas tendrían un estilo más especializado.

De acuerdo con la revisión realizada por Issa (2016: 114-115), podemos resaltar los siguientes rasgos de una PAO:

a) es un género textual planeado y ensayado;

b) no se memoriza ni se lee de un guion;

c) puede tener uno o más oradores;

d) posee un carácter estresante;

e) mejora las habilidades de expresión oral en lengua extranjera, así como dimensiones concretas como la precisión y la fluidez lingüísticas; 
f) habilita al aprendiente para hacer frente a futuros contextos profesionales;

g) tiene una naturaleza compleja;

h) desarrolla y mejora las habilidades tanto críticas como analíticas y agiliza los mecanismos cognitivos.

Teniendo en cuenta los rasgos más sobresalientes del género discursivo de la PAO, consideramos pertinente hacer mención a la siguiente definición de Villar (2014: 49):

Género discursivo oral que tiene como macropropósito informar y/o persuadir en el marco de una relación asimétrica, configurada esencialmente entre un hablante o emisor -el estudiante- y un receptor o destinatario primario -el/la docente-. Asimismo, la audiencia incluye otros destinatarios -los estudiantes que integran el curso- y que se encuentran en relación de simetría con el emisor.

Además, la autora integra los siguientes criterios en la descripción sistemática del concepto de PAO en el contexto académico:

a) posee un carácter predominante expositivo-explicativo y argumentativo;

b) puede pertenecer al ámbito especializado, teórico-científico;

c) depende de un texto escrito (guiones, resúmenes y esquemas, entre otros);

d) tiene una duración definida; y

e) suele incluir un epílogo con turno de preguntas.

A partir de la revisión bibliográfica expuesta, proponemos la siguiente definición para el género textual de la PAO: es un género textual oral, heterogéneo y versátil, en el que interviene, por un lado, el orador -que participa en forma de monólogo- o varios oradores -cuya comunicación puede ser secuencial o interactiva- y, por otro lado, la audiencia, que puede actuar en calidad de lego, semilego o experto; cumple una macrofunción y se adapta a diferentes ámbitos de comunicación: investigación, profesión, formación (tanto en L1 como en L2) y divulgación; el ámbito en el que se produce, la actividad semiótica que desempeña y el propósito comunicativo son determinantes para definir la estructura global del género, así como el orden de secuenciación textual del mismo. En el siguiente apartado profundizamos en la estructura global de la PAO, objeto del presente trabajo.

\section{LA MACROESTRUCTURA DE LA PRESENTACIÓN ACADÉMICA ORAL}

Autores como Zareva (2009a, 2009b) y Villar (2014) destacan el carácter monologado e informacional y la estructura narrativa como características generales de la PAO. Sin embargo, es imprescindible atender a las caracte- 
rísticas macroestructurales de las que este género goza, que varían en cada cultura académica. Se trata de una tarea no del todo fácil, ya que la identificación de las convenciones culturales resulta confusa cuando se realizan estudios contrastivos entre lenguas afines (Ainciburu y Vergara 2015; Calvi 2004; Robles Garrote 2013a, 2013b; Villar y Ainciburu 2013). Villar (2014: 47-49), respaldada por la bibliografía que presenta sobre el tema, considera que la estructura de la PAO en diferentes modelos es una estructura tripartita propia del discurso expositivo y propone la caracterización preliminar de cada una de las secciones y subsecciones de la PAO: apertura del discurso, introducción o exordio, desarrollo de las ideas y epílogo. Estas secciones poseen movimientos o subpartes que se caracterizan por su variabilidad y escasa linealidad, ya que dependen de los propósitos comunicativos que va adquiriendo el discurso. Zareva (2009b), por su parte, sostiene que la PAO adopta diferentes formas y tamaño en términos de longitud, público, objetivos y relación con otros géneros dentro del ámbito académico (clases magistrales, conferencias, tesis y artículos de investigación, entre otros).

No hay suficientes estudios empíricos que hayan estudiado la estructura global de la PAO, y aún menos en EL2, lo que dificulta la anticipación de formatos de estructuras textuales definitivas. No obstante, sí se ha analizado la estructura global del género de la conferencia (Alcaraz Varó 2000; Cieri, Provensal, Bina, Piquer y Sánchez-Centeno 2011; Robles Garrote 2013a, 2013b, 2014; Sanz 2005) y de la PAO en español (Villar 2011, 2014). Además, muchos tutoriales y guías diseñadas por universidades internacionales $^{2}$, en sus espacios dedicados al asesoramiento sobre escritura y oralidad académica, orientan a los estudiantes universitarios sobre diferentes aspectos textuales vinculados a los textos académicos y, de igual modo, respecto a la estructura global de una PAO. En ellos se incide en la importancia de la planificación, el ensayo y la escritura de un guion antes de que se realice el texto oral, destacando tres aspectos:

1) prepararse para la PAO,

2) diseñar y estructurar la PAO, y

3) atender a otros aspectos.

En lo que respecta a la preparación para la $\mathrm{PAO}$, se recomienda la realización de las siguientes tareas:

a) Analizar la futura audiencia, es decir, identificar cuál es su nivel de especialización y cuáles son sus necesidades y demandas.

${ }^{2}$ El Servicio de Información de la Staffordshire University; el Servicio de Apoyo al Estudiante (Student Support Service) de la University of East Anglia; el Servicio de Aprendizaje Efectivo para el Desarrollo de las Habilidades Académicas (Effective Learning Service) de la University of Bradford; el Centro de Ayuda para el Desarrollo de las Habilidades Académicas en la Deakin University (Academic Skills Deaking Study Support); la guía de habilidades orales académicas de la Griffith University y la UNSW Sydney, ambas en Australia. 
b) Presentar los objetivos de la PAO.

c) Buscar información sobre el tema de la PAO.

d) Evaluar las fortalezas y debilidades que ofrecen los materiales aportados.

e) Organizar el material investigado y redactar un borrador con las ideas principales.

f) Tener en cuenta el factor tiempo.

g) Conocer las características del lugar donde se realizará la PAO y preparar apoyo multimedia, si fuera necesario.

En lo que concierne al diseño y estructuración de la PAO, los tutoriales y guías de diseño de la PAO inciden en las tres secciones generales de introducción, cuerpo y conclusión, y la bibliografía consultada muestra un evidente consenso en cuanto a estas secciones:

1) Introducción: incluye la definición de los conceptos o términos clave y un guion o resumen de las ideas generales de la PAO. Los diferentes modelos propuestos por las universidades internacionales anteriormente referidas ofrecen a sus estudiantes modelos de estructura global del género de la PAO y presentan un gran abanico de subsecciones:
a) saludo;
b) autopresentación;
c) enunciación del tema;
d) captación de la atención de la audiencia;
e) presentación de los objetivos de la PAO.

2) Cuerpo: desarrolla los puntos más relevantes del tema de la PAO de forma estructurada, coherente y cronológica, aportando explicaciones y ejemplos si es necesario. Normalmente, la presentación de las ideas principales se realiza mediante el método sándwich o el método a pila ${ }^{3}$.

3) Conclusión: al igual que en la sección de la introducción, se observa una numerosa cantidad de subsecciones diferentes, entre otras:

a) deseo de haber alcanzado el objetivo de la PAO propuesto;

b) mención a los temas que no se han podido abordar;

c) realización de un resumen o síntesis;

d) posibilidad de que el público participe con sus opiniones e impresiones sobre el tema presentado en un turno de preguntas.

${ }^{3}$ El método sándwich consiste en el planteamiento de cada una de las nuevas ideas en tres pasos: (1) se enuncia una idea, (2) se propone la contraargumentación y (3) se presenta la conclusión. Por otro lado, en el método a pila primero se presentan todas las ideas, después, las contraargumentaciones y, finalmente, las conclusiones de cada idea. 
Por último, en lo referente a los demás aspectos adicionales a los que hay que atender en la realización de la PAO, podemos destacar los siguientes:

a) Las características del habla (la voz, la dicción, el uso del humor y de las notas o las diapositivas).

b) El lenguaje corporal correcto: la sonrisa, el entusiasmo, el contacto visual con la audiencia, una postura relajada y con la cabeza erguida mirando a la audiencia.

c) El lenguaje corporal incorrecto: tocarse o taparse la cara, mantener los puños cerrados, colocar las manos en las caderas, tener una expresión seria o deprimida, tener las manos en los bolsillos, ciertos movimientos con las manos y risas innecesarias.

d) Control de los nervios y la ansiedad; para ello, se recomienda:

1) conocer a la audiencia y el género discursivo oral;

2) ser consciente del factor tiempo;

3) comenzar y finalizar con una frase perfectamente clara y centrada en el tema;

4) no tener miedo a ser repetitivo;

5) ensayar la PAO.

e) Manejo del turno de preguntas: estar bien preparado, mantener el contacto visual con la persona que pregunta y responder a la audiencia.

El análisis de las subsecciones de la PAO nos lleva a considerar acertado el hecho de que este género textual adquiera una macroestructura diferente según el ámbito académico en el que se realice (formativo, de divulgación académica y de discusión científica e investigación). El ámbito académico, unido a otros aspectos vinculados a la PAO, tales como el tema tratado en la presentación, influyen en el contenido y en la duración de esta, lo cual a su vez puede condicionar la aparición o no de ciertas subsecciones. Dada la multiplicidad de elementos que pueden condicionar la caracterización del género de la PAO en el ámbito universitario, y por tanto de las subsecciones de la misma, el presente estudio plantea la posibilidad de establecer la aparición o no de estas subsecciones como un elemento opcional, tal y como lo propone Villar (2014).

\section{Metodología}

\subsection{Objetivos del estudio}

Considerando el marco teórico expuesto, los objetivos del estudio que aquí se presenta son: 
1) examinar la estructura global de las PAO realizadas por estudiantes estadounidenses de EL2 del nivel B2 (B2.1 y B2.2) e identificar $\mathrm{y}$ analizar la recurrencia de las secciones generales y subsecciones de estas PAO;

2) ofrecer una propuesta de estructura global de la PAO en EL2 tomando como punto de referencia el modelo de Villar (2014).

\subsection{Informantes y corpus}

El corpus analizado en el presente estudio proviene de 20 informantes, 8 hombres y 12 mujeres, que realizaban cursos de español en el Centro de Estudios Hispánicos de la Universidad Antonio de Nebrija (Madrid) en un contexto de inmersión. Todos ellos eran estadounidenses, de edades comprendidas entre los 19 y los 21 años y procedentes de diferentes universidades y grados.

El corpus estuvo formado por $20 \mathrm{PAO}$ monologadas realizadas en dos cursos: Español de la Empresa y Español en los Medios de Comunicación. Sendos cursos requieren un nivel lingüístico B2 según el Marco Común Europeo de Referencia para las lenguas (MCER; Consejo de Europa 2002). Los temas de las PAO, que fueron asignados previamente por el profesor, y el tiempo requerido para su realización, se recogen en las Tablas 1 y 2 respectivamente.

TABLA 1. Temas de las PAO

\begin{tabular}{lcl}
\hline \multicolumn{1}{c}{ Tema de la PAO } & $\begin{array}{c}\text { Número } \\
\text { de PAO }\end{array}$ & \multicolumn{1}{c}{ Curso } \\
\hline Temas sobre márquetin estratégico & 16 & Español de la Empresa B2.1 \\
\hline $\begin{array}{l}\text { Temas sobre medios } \\
\text { de comunicación }\end{array}$ & 3 & $\begin{array}{l}\text { Español en los Medios } \\
\text { de Comunicación B2.2 }\end{array}$ \\
\hline Descripción de una empresa española & 1 & Español de la Empresa B2.1 \\
\hline
\end{tabular}

TABLA 2. Tiempo asignado para las PAO

\begin{tabular}{lcl}
\hline \multicolumn{1}{c}{ Tema de la PAO } & Tiempo & \multicolumn{1}{c}{ Curso } \\
\hline Temas sobre márquetin estratégico & 10 minutos & Español de la Empresa B2.1 \\
\hline $\begin{array}{l}\text { Temas sobre medios } \\
\text { de comunicación }\end{array}$ & $\begin{array}{c}\text { No } \\
\text { especificado }\end{array}$ & $\begin{array}{l}\text { Español en los Medios } \\
\text { de Comunicación B2.2 }\end{array}$ \\
\hline Descripción de una empresa española & 10 minutos & Español de la Empresa B2.1 \\
\hline
\end{tabular}




\subsection{Procedimiento}

Las PAO pertenecen a una tarea de evaluación parcial que se llevó a cabo en una sesión de clase. Cabe señalar que los estudiantes no recibieron instrucción previa sobre la estructura global de la PAO que debían seguir. Todas las PAO se videograbaron y su transcripción se hizo en formato CHAT (Programas CLAN; MacWhinney 2000). Para el análisis de la macroestructura de las PAO se partió del modelo de Villar (2014), siguiendo las pautas proporcionadas por la autora sobre las posibles secciones y subsecciones que debe contener una PAO.

\section{RESUlTADOS Y DisCUSIÓN}

La Tabla 3 muestra el análisis de la macroestructura de las 20 PAO monologadas. Los resultados evidencian un uso individual en la aparición de las secciones y subsecciones de las PAO. Siguiendo el modelo de Villar (2014), se ha observado que en todas las PAO analizadas aparecen las cuatro secciones fundamentales:

1) «Apertura del discurso» (Sección 1, S1);

2) «Introducción al tema» (Sección 2, S2);

3) «Desarrollo del tema» (Sección 3, S3); y

4) «Epílogo» (Sección 4, S4).

Este modelo se asemeja a la estructura general que promueven las guías y manuales publicados por universidades internacionales y otros autores anteriormente mencionados, quienes establecen que una PAO debe contener al menos tres bloques: «Introducción» (que abarca las S1 y S2), «Cuerpo» (S3) y «Conclusión» (S4). Por tanto, se podría afirmar que los informantes del presente estudio son conscientes de las secciones universales que debe contener este género discursivo tal vez debido a la instrucción previa que hubieran recibido sobre la $\mathrm{PAO}$ o géneros discursivos en general en su L1 y en el marco de sus estudios universitarios.

No obstante, el análisis de las PAO también mostró un uso idiosincrático de las subsecciones propuestas por Villar (2014), al igual que otras propuestas de macroestructura del género discursivo oral. En concreto, se han identificado subsecciones que fueron utilizadas por un gran número de estudiantes tales como el «Saludo» en la S1 (9 PAO), la «Enunciación del tema» en la S2 (19 PAO), la «Conclusión» (9 PAO), el «Cierre del expositor» (14 PAO), la «Apertura del turno de preguntas» (11 PAO) y la «Discusión» (11 PAO) en la S4. 


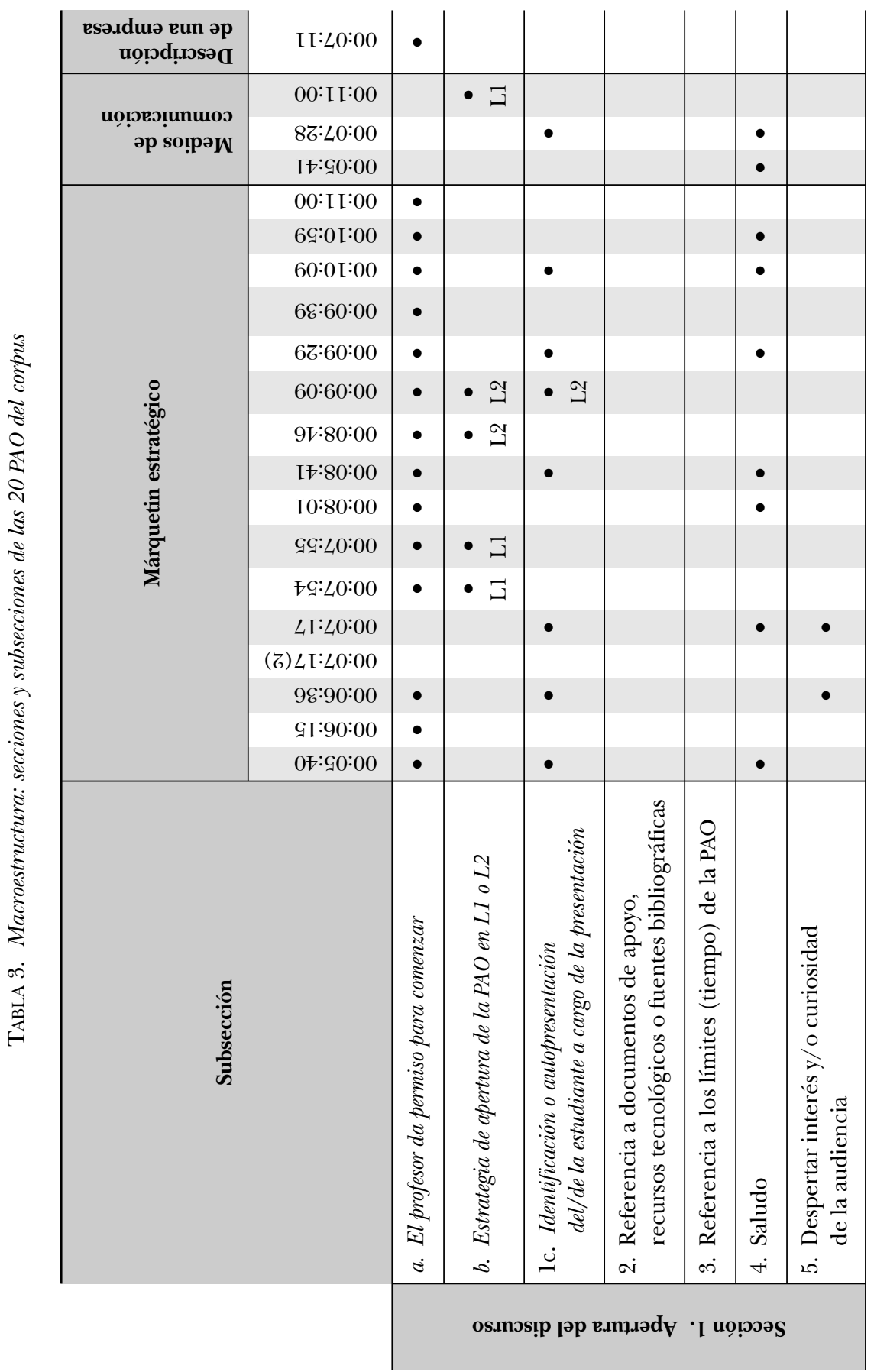




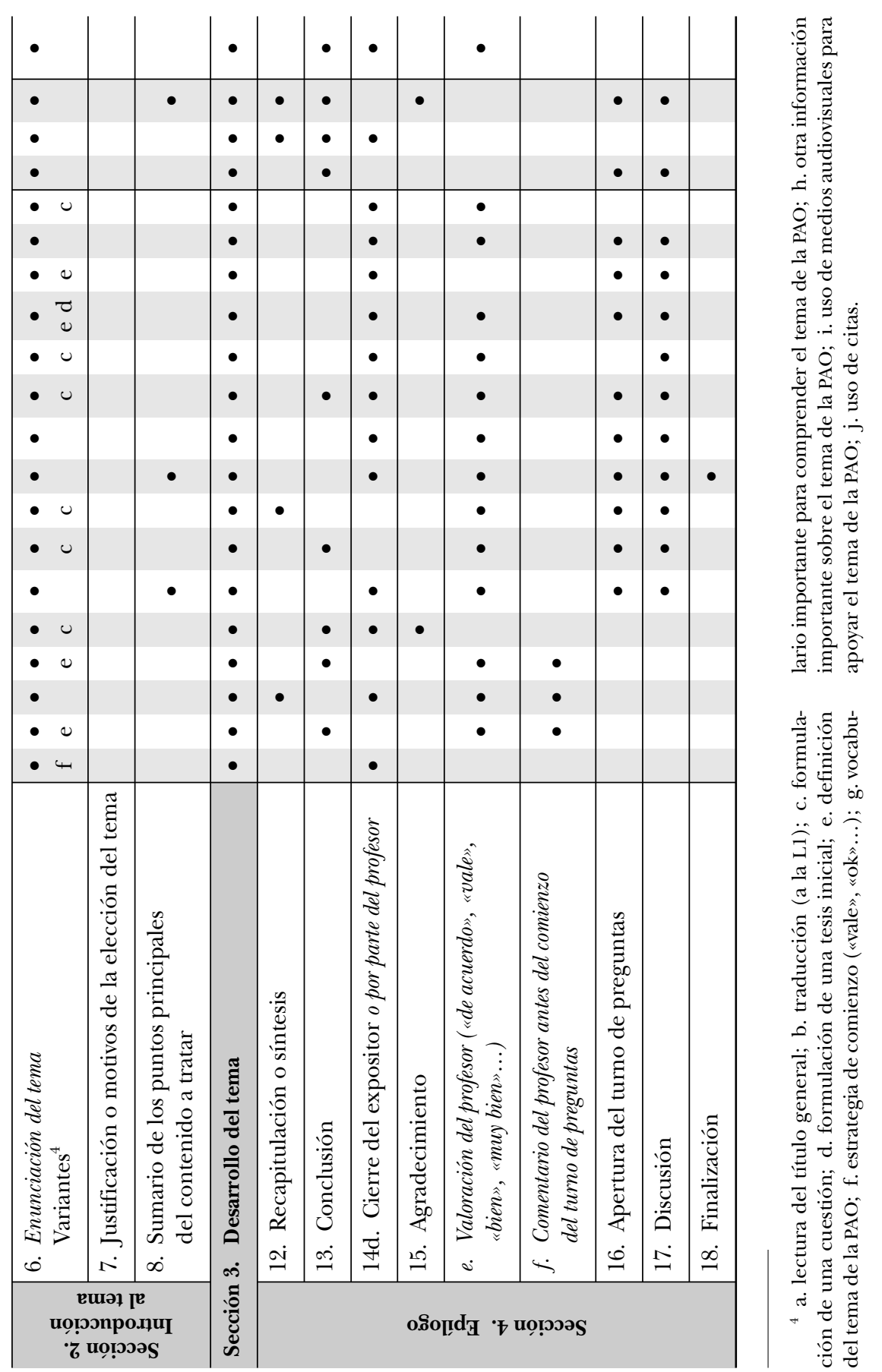


En cuanto al «Saludo» (S1), los informantes del presente estudio utilizaron en un $45 \%$ esta subparte. Este resultado contradice los de Villar (2014), que revelaron un uso minoritario del saludo en las PAO de aprendientes alemanes de español en comparación con las PAO de estudiantes alemanes nativos, quienes sí utilizaron el saludo como forma ritualizada para comenzar un discurso al público. Por otro lado, la subsección de «Enunciación del tema» (S2) fue utilizada por todos los participantes, y este hallazgo se asemeja a los obtenidos por Villar (2014). Podría sostenerse que dicha subsección marca el comienzo del cuerpo de la PAO y, por tanto, su presencia adquiere un carácter obligatorio.

Por lo que respecta a la «Conclusión» (S4), tiene una presencia en el corpus del $45 \%$, porcentaje que debe considerarse junto con el de la subsección de «Recapitulación o síntesis» $(20 \%)$. Dichas subsecciones se emplean con el objetivo de recopilar las ideas principales del cuerpo de la PAO, aunque estimaríamos que el aprendiente de EL2 no discierne entre el significado de ambas secciones.

A diferencia del amplio uso de las subsecciones descritas anteriormente, los resultados del presente estudio evidencian la ausencia o limitada utilización de otras subsecciones como «Referencia a documentos de apoyo, recursos tecnológicos o fuentes bibliográficas», «Referencia a los límites (tiempo) de la $\mathrm{PAO}$ » $\mathrm{y}$ «Despertar interés y/o curiosidad de la audiencia» en la S1, «Justificación o motivos de la elección del tema» y «Sumario de los puntos principales del contenido a tratar» en la S2, mientras que en la S4 observamos la precaria utilización de las subsecciones «Recapitulación o síntesis» $\mathrm{y}$ «Agradecimiento».

En concreto, ninguno de los informantes del presente estudio utilizó la subsección «Referencia a documentos de apoyo, recursos tecnológicos o fuentes bibliográficas». Este resultado está en consonancia con los obtenidos por Villar (2014) en cuanto al corpus de las PAO realizadas por los aprendientes alemanes de español que participaron en su estudio, a diferencia de las PAO nativas alemanas en las que dicha subsección estuvo más presente. En la revisión de materiales y guías para la elaboración de PAO publicadas por universidades de EE.UU., Reino Unido y Australia no se evidencia ninguna subsección dirigida a este fin, lo que podría justificar la limitada presencia de este contenido en las PAO procedentes de la cultura académica estadounidense, en general, y en las PAO de los estudiantes estadounidenses del presente estudio, en particular. Dada esta circunstancia, se podría argumentar sobre la opcionalidad de esta subsección en una propuesta de macroestructura de la PAO para aprendientes de EL2, ya que el contenido de esta subsección parece estar influenciado por cuestiones relacionadas con la cultura idiosincrásica del país donde se realicen estudios de este tipo. 
La subsección «Referencia a los límites (tiempo) de la PAO» tampoco fue utilizada por ninguno de los informantes del estudio. El trabajo de Villar (2014) también mencionaba un uso minoritario de esta subsección en las PAO de español (L1 alemán) analizadas en su investigación. A tenor de los resultados obtenidos, de nuevo se podría asumir el carácter opcional de esta subsección y su potencial inclusión en una PAO en función de la cultura académica del estudiante o ponente.

En lo que respecta a la subsección «Despertar interés y/o curiosidad de la audiencia» en la S1, y al contrario de lo que promulgan los manuales sobre elaboración de PAO consultados, los resultados del presente estudio parecen indicar una posible carencia de instrucción previa o una instrucción limitada en cuanto al uso de esta subsección (encontrada en tan solo 2 PAO).

Respecto a la subsección «Justificación o motivos de la elección del tema», no apareció en ninguna PAO del corpus. Dicha ausencia puede estar suscitada por el hecho de que el tema de las PAO analizadas había sido previamente asignado por el profesor, por lo que hacer referencia a esta información resultaría repetitivo.

En lo que concierne a la subsección «Sumario de los puntos principales del contenido a tratar», resulta relevante señalar que apareció en tan solo 3 PAO, mientras que en el estudio de Villar (2014) se utilizó de forma mayoritaria por el grupo de estudiantes alemanes de español. Tal diferencia podría atribuirse a diferencias entre las dos culturas académicas estudiadas, la alemana y la estadounidense, así como a la falta de instrucción previa relativa a la macroestructura de la PAO.

$\mathrm{Al}$ igual que con la subsección descrita anteriormente, la «Recapitulación o síntesis» fue utilizada en solo 4 casos. Este uso limitado es también marcado en los resultados de Villar (2014) y podría deberse a que esta subsección suele asociarse erróneamente a la sección de «Conclusión». Finalmente, en cuanto a la subsección de «Agradecimiento» se encontró en solo 2 PAO del corpus. Entendemos que esta circunstancia se ve motivada por el hecho de que, una vez más, el discente no ha recibido instrucción previa para hacerlo.

\subsection{Nuevas subsecciones}

Después de haber realizado el recuento y análisis de las secciones y subsecciones del corpus de PAO, este estudio identificó nuevas subsecciones a partir del modelo de Villar (2014) (señaladas en cursiva en la Tabla 3) que se detallan a continuación:

- a. El profesor da permiso para comenzar. Esta intervención del profesor nos lleva a considerar la importancia de la labor del docente al ini- 
cio de una PAO en L2, por lo que podría constituir una firme candidata a formar parte de la S1 de la PAO académica. Si bien es cierto que la mediación del profesor puede adquirir un carácter individual y propio de cada docente, esta circunstancia ya es apreciada por Villar (2014).

- $\quad$ b. Estrategia de apertura de la PAO en L1 o L2. Se observó de manera escasa, pero presente, que 5 informantes del presente estudio hicieron uso de una estrategia para iniciar la PAO que consiste en el uso de palabras y elementos léxicos en su L1 o en EL2 (por ejemplo, muletillas del tipo well, ok,...).

- $\quad$ 1c. Identificación o autopresentación del/de la estudiante a cargo de la presentación. Los resultados mostraron 8 casos de PAO en los que aparece una autopresentación del ponente, aspecto probablemente vinculado a la cultura norteamericana.

- 6. Enunciación del tema. Esta subsección, presente en 19 de las 20 PAO del corpus analizado, ya había sido mencionada por Villar (2014), quien propone cuatro variantes para enunciar el tema de la PAO: lectura del título general, traducción a la L1, formulación de una cuestión y formulación de una tesis inicial. Además de estas variantes, este trabajo plantea las siguientes seis variantes adicionales:

1) definición del tema de la PAO;

2) estrategia de comienzo («vale», «ok», etc.);

3) vocabulario importante para comprender el tema de la PAO;

4) otra información importante sobre el tema de la PAO;

5) uso de medios audiovisuales para apoyar el tema de la PAO;

6) uso de citas.

- $\quad 14 d$. Cierre del expositor o por parte del profesor. En relación con esta subsección, consideramos oportuno incorporar la figura del profesor como agente que, además del estudiante-ponente, puede llevar la presentación a su fin.

- e. Valoración del profesor («de acuerdo», «vale», «bien», «muy bien»...). Dicha subsección presenta la intervención del docente para realizar una valoración general sobre la presentación expuesta y también es susceptible de una valoración individual.

- $\quad$ f. Comentario del profesor antes del comienzo del turno de preguntas.

\subsection{Propuesta de estructura global de la PAO en EL2}

Fruto de los resultados obtenidos en la presente investigación, especialmente de la identificación de las nuevas subsecciones que podrían formar parte de un modelo de PAO en el ámbito académico-universitario, plan- 
teamos en la Tabla 4 una propuesta de estructura global de la PAO para el aula de EL2 que comprende cuatro secciones generales y comunes a todas las PAO analizadas y 20 subsecciones complementarias. Dicha propuesta difiere de la presentada por Villar (2014: 135-138) en los siguientes aspectos:

a) El carácter opcional u obligatorio de algunas subsecciones:

- La estructura global de la PAO para aprendientes de EL2 propuesta en la presente investigación comprende 7 subsecciones de carácter obligatorio. En concreto, considera como obligatorias las subsecciones de «Saludo» (S1), «Identificación o autopresentación del/de la estudiante a cargo de la presentación» (S1), «Identificación del tema» (S1) y «Cierre por parte del expositor o del profesor» (S4), a diferencia de Villar (2014), que las considera opcionales.

- Las subsecciones de la nueva propuesta de estructura global de la PAO que poseen un carácter opcional son 13. Estas pueden adherirse a la naturaleza de la PAO; en caso de que no se produjesen, no dificultaría la progresión narrativa de la PAO.

b) La inclusión de nuevas subsecciones (ns) o ampliación del contenido de subsecciones (ac) anteriormente propuestas (Villar 2014).

c) A diferencia de la propuesta de Villar (2014), consideramos que la Sección 3 de «Desarrollo del tema» no puede especificarse con mayor detalle en un modelo de estructura global de la PAO. Esta sección es el cuerpo de la PAO y está determinada por la organización del contenido de la misma. Esta circunstancia condicionaría la tipología textual predominante que se utilizará en la PAO, así como la secuencia completa de movimientos textuales que conformarán esta sección.

TABLA 4. Modelo de estructura global de la PAO para el aula de EL2

\begin{tabular}{cllc}
\hline \multirow{2}{*}{ SECCIÓN } & \multicolumn{1}{c}{ SUBSECCIONES } & CARÁCTER \\
\hline & El profesor da permiso para comenzar (ns) & Opcional \\
\cline { 2 - 3 } & Estrategia de apertura de la PAO en L1 o L2 (ns) & Opcional \\
\cline { 2 - 3 } \begin{tabular}{c} 
Sección 1. $\begin{array}{c}\text { Apertura } \\
\text { del discurso }\end{array}$ \\
\cline { 2 - 3 }
\end{tabular} & $\begin{array}{l}\text { Saludo } \\
\text { Identificación o autopresentación del/de la }\end{array}$ & Obligatorio \\
\cline { 2 - 3 } & estudiante a cargo de la presentación $(\mathrm{ac})$ & Obligatorio \\
\hline
\end{tabular}


TABLA 4. (cont.)

\begin{tabular}{|c|c|c|}
\hline SECCIÓN & SUBSECCIONES & CARÁCTER \\
\hline \multirow{3}{*}{$\begin{array}{c}\text { Sección } 1 . \\
\text { Apertura } \\
\text { del discurso } \\
\text { (cont.) }\end{array}$} & $\begin{array}{l}\text { Referencia a documentos de apoyo, recursos } \\
\text { tecnológicos o fuentes bibliográficas }\end{array}$ & Opcional \\
\hline & Referencia a los límites (de tiempo) de la PAO & Opcional \\
\hline & Despertar interés y/o curiosidad de la audiencia & Opcional \\
\hline \multirow{13}{*}{$\begin{array}{l}\text { Sección } 2 . \\
\text { Introducción } \\
\text { al tema }\end{array}$} & Enunciación del tema & \\
\hline & Variantes: & \\
\hline & a. Lectura del título general & \\
\hline & b. Traducción (a la L1) & \\
\hline & c. Formulación de una cuestión & \\
\hline & d. Formulación de una tesis inicial & \\
\hline & e. Definición del tema de la PAO (ac) & Obliontorio \\
\hline & $\begin{array}{l}\text { f. Estrategia de comienzo («vale», «ok»...) (ac) } \\
\text { g. Vocabulario importante para comprender } \\
\text { la PAO (ac) }\end{array}$ & Obligatorio \\
\hline & h. Otra información importante (ac) & \\
\hline & $\begin{array}{l}\text { i. Uso de medios audiovisuales para apoyar } \\
\text { el tema de la PAO (ac) }\end{array}$ & \\
\hline & j. Uso de citas (ac) & \\
\hline & Justificación o motivos de la elección del tema & Opcional \\
\hline & $\begin{array}{l}\text { Sumario de los puntos principales del contenido } \\
\text { a tratar }\end{array}$ & Opcional \\
\hline \multicolumn{2}{|c|}{ Sección 3. Desarrollo del tema } & Obligatorio \\
\hline \multirow{9}{*}{$\begin{array}{l}\text { Sección } 4 . \\
\text { Epílogo }\end{array}$} & Recapitulación o síntesis & Opcional \\
\hline & Conclusión & Obligatorio \\
\hline & Cierre del expositor o por parte del profesor (ac) & Obligatorio \\
\hline & Agradecimiento & Opcional \\
\hline & $\begin{array}{l}\text { Valoración del profesor («de acuerdo», «vale», } \\
\text { «bien», «muy bien»...) (ns) }\end{array}$ & Opcional \\
\hline & $\begin{array}{l}\text { Comentario del profesor antes del comienzo } \\
\text { del turno de preguntas (ns) }\end{array}$ & Opcional \\
\hline & Apertura del turno de preguntas & Opcional \\
\hline & Discusión & Opcional \\
\hline & Finalización & Obligatorio \\
\hline
\end{tabular}




\section{Conclusiones}

El presente estudio ha permitido arrojar luz acerca del uso real e irregular de las secciones y subsecciones que conforman la estructura global de la PAO en el contexto formativo. En concreto, se pudo constatar las secciones generales de la PAO, al igual que lo atestigua la investigación secundaria, pero también el carácter dinámico de sus subsecciones y su alta capacidad de adaptabilidad y versatilidad a diferentes ámbitos de la vida académica. Estas circunstancias nos llevaron a las siguientes conclusiones: en primer lugar, no es posible diseñar un único modelo de estructura global de la PAO; en segundo lugar, la estructura de la PAO está vinculada al ámbito donde aparece, ya sea el académico de orientación, asesoramiento y del aula, o el ámbito académico de investigación y divulgación.

Según los resultados de investigaciones empíricas previas sobre análisis de géneros académicos desde una perspectiva contrastiva entre diferentes culturas académicas y entre lenguas afines y no afines (Ainciburu y Vergara 2015; Villar 2014; Villar y Ainciburu 2013), las PAO se caracterizan por su carácter narrativo e informacional, su naturaleza dialógica, su densidad léxica, y la macroestructura de la PAO. Además, los diferentes arquetipos culturales propician un carácter diferente en las PAO; por ejemplo, mientras que las PAO alemanas y estadounidenses en los estudios citados suelen poseer un carácter dialógico, las PAO italianas son monologadas.

Las PAO analizadas en esta investigación presentan un carácter monologado, aunque 11 de ellas muestran cómo la subsección «Discusión» da pie a que este carácter evolucione hacia un carácter dialogado. Esta variedad de caracteres nos instaría a incidir una vez más en el carácter dinámico de la PAO, que está inmerso en un continuo de posibilidades que van desde aquella PAO que es estrictamente monologada a aquella que es dialogada. De este modo, este estudio propone que el dinamismo del carácter académico de la PAO estaría propiciado por:

- El ámbito de socialización académica donde se ubica la PAO:

1) de orientación, de asesoramiento y del aula;

2) institucional;

3) de investigación y divulgación.

- La utilización de la PAO como:

1) pre-tarea, tarea principal o post-tarea;

2) herramienta de evaluación (parcial o final).

- El grado de intervención del docente: al principio, durante o al final de la PAO.

- La existencia o no de la subsección «turno de preguntas», causa que motiva la mutación de una PAO monologada en una dialógica. 
Este trabajo replantea la estructura global de la PAO propuesta por Villar (2014) y presenta una macroestructura de este género dirigida al ámbito del aula o formativo en EL2. Dicha macroestructura mantiene las 4 secciones generales y propone 20 subsecciones. De ellas, 7 poseen un carácter obligatorio y 13 subsecciones son de carácter opcional, es decir, son susceptibles de adherirse a la PAO pero, en caso contrario, no dificultarían su progresión. Finalmente, es posible afirmar que el carácter opcional de las subsecciones depende no solamente de factores culturales, sino también de variables que vinculan el género discursivo con el ámbito en el que se realiza y de las instrucciones previamente recibidas. 


\section{BIBLIOGRAFÍA}

Ainciburu, María Cecilia y María Ángeles Vergara (2015): «Las presentaciones orales en la evaluación del español académico. Las concepciones previas del docente y la actuación de alumnos universitarios de diferentes nacionalidades». En M. A. Lamolda y O. Cruz (eds.), La formación y competencias del profesorado de ELE, Actas del XXVI Congreso Internacional ASELE, Granada: Universidad de Granada, 1029-1037. <https://cvc.cervantes.es/ensenanza/ biblioteca_ele/asele/pdf/26/26_1029.pdf>.

Alcaraz VAró, Enrique (2000): El inglés profesional y académico, Madrid: Alianza.

- (2007): «La sociedad del conocimiento, marco de las lenguas profesionales y académicas». En E. Alcaraz Varó, J. Mateo Martínez y F. Yus Ramos (eds.), El inglés profesional y académico, Madrid: Alianza, 3-11.

Andrade-Calderón, Martha Cecilia y Clarena Muñoz-Dagua (2014): «Las fórmulas retóricas del rebusque. Un estudio desde la semiótica de Halliday», Tabula Rasa 20, 329-345. <http://www.scielo.org.co/pdf/tara/n20/n20a14.pdf>.

BajTín, Mijaíl (1982): Estética de la creación verbal, Madrid: Siglo XXI Editores.

CALVI, María Victoria (2004): «Aprendizaje de lenguas afines: español e italiano», Revista RedELE 1. <https://www.mecd.gob.es/dam/jcr:c99a48e2-7e87-4cbe9de6-94ff9458912a/2004-redele-1-02calvi-pdf.pdf $>$.

Ciapuscio, Guiomar Elena (1994): Tipos textuales, Buenos Aires: Universidad de Buenos Aires, Instituto de Lingüística.

Cieri, Lidia Mabel, Laura Provensal, Eugenia Lucía Bina, Verónica PiQuer y Adelina SÁnchez-Centeno (2011): «Audio-Comprensión de conferencias en inglés en distintas áreas de estudio", Revista Contextos de Educación 11. $<$ http://www.hum.unrc.edu.ar/publicaciones/contextos/articulos/2011/pdfs/ 07-cieri.pdf $>$.

Consejo de EuRoPa (2002): Marco común europeo de referencia para las lenguas: aprendizaje, enseñanza, evaluación, Madrid: Secretaría General Técnica del MEC, Anaya e Instituto Cervantes. <http://cvc.cervantes.es/ensenanza/biblioteca_ ele/marco/cvc_mer.pdf $>$.

Dolz, Joaquin y Roxane GAGNON (2010): «El género textual, una herramienta didáctica para desarrollar el lenguaje oral y escrito», Lenguaje 38/2, 497-527.

GARCíA-IZQUIERDO, Isabel (2007): «Los géneros y las lenguas de especialidad (I)». En E. Alcaraz Varó, J. Mateo Martínez y F. Yus Ramos (eds.), El inglés profesional y académico, Madrid: Alianza, 119-124.

IssA, Ali (2016): "The effects and implications of implementing oral presentations in an Omani ICLHE Classroom», Asian EFL Journal 18/2, 113-155.

MaCWhinNeY, Brian (2000): The CHILDES Project. Tools for analysing talk (3 $3^{\mathrm{a}} \mathrm{ed}$.), Mahwah, NJ: Lawrence Erlbaum Associates.

MaURANEN, Anna (2007): «Investigating English as a lingua franca with a spoken corpora». En M. C. Campoy y M. J. Luzón (eds.), Spoken corpora in Applied Linguistics, Berlin: Peter Lang, 33-56. 
Muñoz-Dagua, Clarena, Martha Cecilia Andrade-Calderón y Mireya CisnerosEsTUPIÑÁn (2015): «Los indicios de la actitud en las interacciones orales en el aula universitaria», Folios 42, 127-138 (doi:10.17227/01234870.42folios127.138).

Regueiro, María Luisa y Daniel Sáez (2013): El español académico. Guía práctica para la elaboración de textos académicos, Madrid: Arco/Libros.

Robles GARRote, Pilar (2013a): «La conferencia como género monológico: análisis macroestructural en español e italiano», Boletín de Filología 48(1), 127-146. $<$ http://www.boletinfilologia.uchile.cl/index.php/BDF/article/viewFile/271 $66 / 28785>$.

- (2013b): «Retórica contrastiva y enseñanza del discurso formal en lenguas afines», Pragmalingüistica 21, 121-137. <http://revistas.uca.es/index.php/ pragma/article/view/1851>.

- (2014): «Propiedades contextuales del discurso académico-científico: la conferencia y sus variantes», Sintagma 26, 119-131. <http://www.sintagma.udl.cat/ export/sites/Sintagma/documents/articles_26/robles.pdf >.

RojAS-GARCíA, Ilene (2016): «Aportes de la lingüística sistémico-funcional para la enseñanza de la lectura y la escritura en la educación superior», Educación y Educadores 19/2, 185-204. <http://educacionyeducadores.unisabana.edu.co/ index.php/eye/article/view/4831/4279>.

SANZ, María Inmaculada (2005): El español profesional y académico en el ámbito de la ingeniería civil. El discurso oral y escrito (Tesis doctoral), Valencia: Universidad de Valencia. <http://www.tdx.cat/handle/10803/9817>.

SWALES, John (1990): Genre analysis: English in academic and research settings, Cambridge: Cambridge University Press.

van DIJK, Teun (1978): La ciencia del texto. Un enfoque interdisciplinario, Barcelona: Paidós.

VÁzQUEZ, Selene (2016): «La presentación oral en ELE. Hacia un modelo didáctico para su enseñanza en contexto escolar». En E. Tobar y M. E. Mañas (eds.), El español como lengua extranjera en Portugal II: Retos de la enseñanza de lenguas cercanas, Madrid: Ministerio de Educación, Cultura y Deporte, 186-196.

VILLAR, Claudia Mariela (2011): «Las presentaciones académicas orales de los estudiantes alemanes de E/LE. Del discurso monológico al dialógico», Revista Nebrija de Lingüistica Aplicada a la Enseñanza de Lenguas 10, 130-172. $<$ https://www.nebrija.com/revista-linguistica/files/articulosPDF/articulo_52 98530786f4b.pdf>.

- (2014): Las presentaciones académicas orales en E/LE de estudiantes alemanes, Frankfurt: Peter Lang.

Villar Claudia Mariela y María Cecilia Ainciburu (2013): «Coocurrencia de rasgos lingüísticos en la caracterización de la presentación académica oral EFE», Revista Nebrija de Lingüistica Aplicada a la Enseñanza de Lenguas 13, 30-44. <https:// www.nebrija.com/revista-linguistica/files/articulosPDF/articulo_527761492 f31c.pdf $>$.

ZAREVA, Alla (2009a): «Informational packaging, level of formality, and the use of circumstance adverbials in L1 and L2 student academic presentations», Journal of English for Academic Purposes 8, 55-68 (doi:10.1016/j.jeap.2008.12.002).

- (2009b): «Student academic presentations. The processing side of interactiveness», English Text Construction 2/2, 265-288 (doi:10.1075/etc.2.2.08zar). 